\title{
Thermal Transients Excite Neurons through Universal Intramembrane Mechanoelectrical Effects
}

\author{
Michael Plaksin, ${ }^{1}$ Einat Shapira, ${ }^{1}$ Eitan Kimmel, ${ }^{1}$ and Shy Shoham ${ }^{1,2, *}$ \\ ${ }^{1}$ Faculty of Biomedical Engineering and Russell Berrie Nanotechnology Institute, \\ Technion-Israel Institute of Technology, Haifa 32000, Israel \\ ${ }^{2}$ Department of Ophthalmology and Department of Neuroscience and Physiology, \\ New York University Langone Health, New York, New York 10016, USA
}

(Received 14 March 2017; revised manuscript received 27 November 2017; published 16 March 2018)

\begin{abstract}
Modern advances in neurotechnology rely on effectively harnessing physical tools and insights towards remote neural control, thereby creating major new scientific and therapeutic opportunities. Specifically, rapid temperature pulses were shown to increase membrane capacitance, causing capacitive currents that explain neural excitation, but the underlying biophysics is not well understood. Here, we show that an intramembrane thermal-mechanical effect wherein the phospholipid bilayer undergoes axial narrowing and lateral expansion accurately predicts a potentially universal thermal capacitance increase rate of $\sim 0.3 \% /{ }^{\circ} \mathrm{C}$. This capacitance increase and concurrent changes in the surface charge related fields lead to predictable exciting ionic displacement currents. The new MechanoElectrical Thermal Activation theory's predictions provide an excellent agreement with multiple experimental results and indirect estimates of latent biophysical quantities. Our results further highlight the role of electro-mechanics in neural excitation; they may also help illuminate subthreshold and novel physical cellular effects, and could potentially lead to advanced new methods for neural control.
\end{abstract}

DOI: 10.1103/PhysRevX.8.011043

Subject Areas: Biological Physics,

Computational Physics, Optics

\section{INTRODUCTION}

Optical neurostimulation modalities have gained considerable attention during the past decade as methods for precision perturbation or control of neural activity, primarily as a result of the coemergence of optogenetics [1] and of direct infrared neural stimulation (INS) [2]. Both approaches also offer the long-term prospect of remotely affecting aberrant localized neural circuits that underlie many neurological diseases. A multitude of INS-related studies explored the ability of short-wave infrared (IR) pulses to stimulate neural structures including peripheral $[3,4]$ and cranial nerves [5-10], retinal and cortical neurons [10-12], as well as cardiomyocytes $[13,14]$. It is stipulated that the INS phenomenon is mediated by temperature transients induced by IR absorption [15-17]; such transients can alternatively be induced using other forms of photoabsorption [18-20], or potentially by any other physical form of thermal

\footnotetext{
* Corresponding author. Shoham@nyu.edu

Published by the American Physical Society under the terms of the Creative Commons Attribution 4.0 International license. Further distribution of this work must maintain attribution to the author(s) and the published article's title, journal citation, and DOI.
}

neurostimulation that can be driven rapidly enough $[21,22]$. Shapiro et al. [16] showed that these rapid temperature variations are directly accompanied by changes in the cell membrane's capacitance and resulting displacement currents which are unrelated to specific ionic channels; their findings on the thermal capacitance increase have been supported by experiments from several additional groups [19,20,23-25]. Shapiro et al. [16] also developed a theoretical model where the temperature elevation was seen to give rise to membrane capacitance increase at the membrane's boundary regions (see also Liu et al. [26] and Rabbit et al. [27]). However, as recently pointed out in our reanalysis of this theoretical model $[28,29]$, upon correcting a mathematical convention error it actually predicts a net capacitance decrease, contrary to the experimental measurements.

To address this major apparent gap in the theoretical basis for thermal excitation, we deconstruct and analyze here alternative revised biophysical models (tentative and detailed), where the membrane's physical dimensions themselves also vary in response to the temperature changes, to accurately reflect direct experimental findings [30-33]. The new mechanoelectrical thermal activation (META) models calculate the effect of temperature change on the membrane electrical parameters directly and explicitly (rather than implicitly), and are found to both qualitatively and quantitatively predict empirical findings on thermal membrane 
capacitance increases $[16,19,20,24]$, unraveling the two underlying sources for thermal membrane currents, and making it possible to indirectly estimate from neural stimulation results a significant new quantity, the membrane surface charge difference.

\section{RESULTS}

\section{A. Dimensional changes are crucial for explaining capacitive thermal response}

Capacitive thermal changes were first measured by Shapiro et al. [16] and subsequently by others in artificial membranes [19], human embryonic kidney (HEK) cells [20], and cardiac myocytes [24]. Interestingly, placing these disparate measurements on a uniform capacitancerate scale shows that they all approximately share a universal rate of $\sim 0.3 \% /{ }^{\circ} \mathrm{C}$ (mean $0.29 \pm 0.01 \% /{ }^{\circ} \mathrm{C}$ ), suggesting a potentially universal basis [Fig. 1(a)].

We next examined how these changes compare to the membrane's capacitance thermal rate of change expected purely from temperature-induced dimensional changes, which were recently experimentally estimated using $\mathrm{x}$-ray and neutron small-angle scattering measurements [30-32] and computationally reproduced using molecular dynamics simulations [33]. Using a naive plate capacitor assumption where $C=\left[\left(\varepsilon_{m} A\right) / \delta\right]$, the $0.11 \pm 0.03 \% /{ }^{\circ} \mathrm{C}$ reduction in the phospholipid membrane thickness and $0.22 \pm 0.03 \% /{ }^{\circ} \mathrm{C}$ increase in the area per phospholipid molecule [see Fig. 1(b) and Sec. IV] contribute to a linear increase of $0.33 \pm 0.05 \% /{ }^{\circ} \mathrm{C}$ (relative to the corresponding parameters in $20^{\circ} \mathrm{C}$, neglecting minor thermal variations in the phospholipids' dielectric constant [34]; see Sec. III) putatively explaining the observed capacitance rates [Fig. 1(a)].

\section{B. Detailed biophysical model}

To further understand the impact of this mechanism on membrane currents and its potential for settling the theoretical conceptual gap, we subsequently studied the effect of temperature changes and transients on a detailed GouyChapman-Stern (GCS) [35-39] multicompartment realistic biophysical model of the phospholipid membrane electrochemistry. The various model parameters were taken "as is" without retuning or post hoc adjustments, and are based on known or previously measured physical and biophysical quantities, wherever attainable (summarized in Table SI of Supplemental Material with the respective sources [40]). In the model, the membrane geometry and physical properties are represented by five regions with different characteristics [Fig. 2(a) and Table I]: two bulk regions for each of the membrane's sides (intracellular and extracellular), where the ions' concentrations are spatially Boltzmann distributed, obtained when diffusion and electrical forces on the different ions reach equilibrium, and two Stern layers with thicknesses determined by the phospholipid polar head size and the average effective radius of the ions that are the closest to the membrane and a central hydrophobic region, occupied by the tails of the phospholipid molecules [16,35-39]. The ions in each of the bulk regions are comprised of the mobile and fixed extramembranal charges (intracellular, $Q$ and $-\sigma_{i}$; extracellular, $-Q$ and $-\sigma_{o}$ ); the fixed charges have the same absolute value, but opposite signs as the membrane leaflets' surface charges to which they are electrostatically attracted [Fig. 2(a)]. (a)

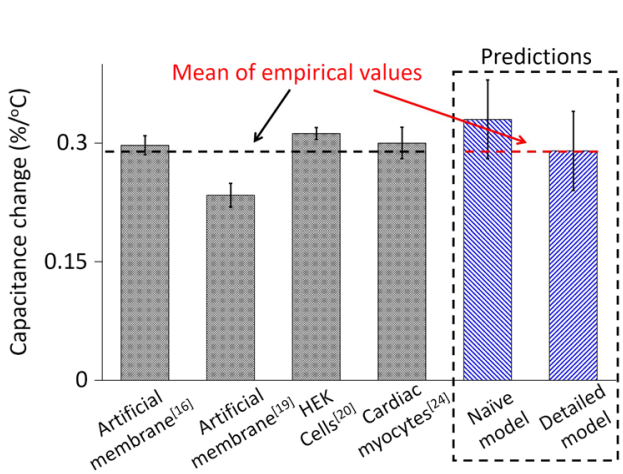

(b)
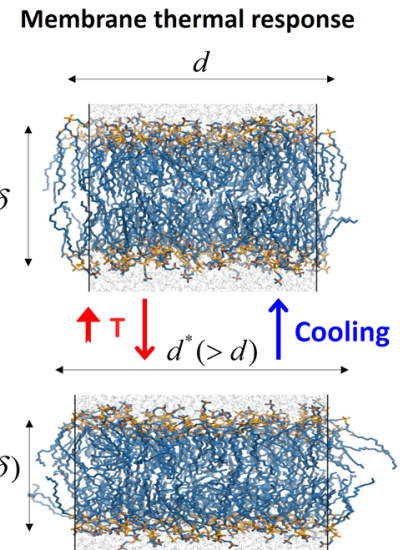

FIG. 1. Universal membrane capacitance thermal response rate, explained by membrane dimensional changes. (a) Membrane capacitance change rates measured in different studies and preparations (gray bars) versus predictions from the thermal dimensional response of POPC membrane bilayers (naive plate capacitor model), and from a detailed biophysical model accounting for spatial charge distribution. (b) Schematized membrane thinning and area expansion under temperature elevation. This observed process [30-33] is thought to result from an increase in the phospholipid molecules' fatty acid chains trans-gauche rotational isomerization, which shortens the tails' effective length and increases the area per phospholipid molecule. Biophysically, these two phenomena contribute to a predictable increase in both the membrane hydrophobic core's and total capacitance. Error bars for the direct capacitance measurements are \pm s.e.m, and for the model predictions are \pm chi-squares distribution-related uncertainty [31]. 
(a)

\section{Detailed biophysical membrane model}

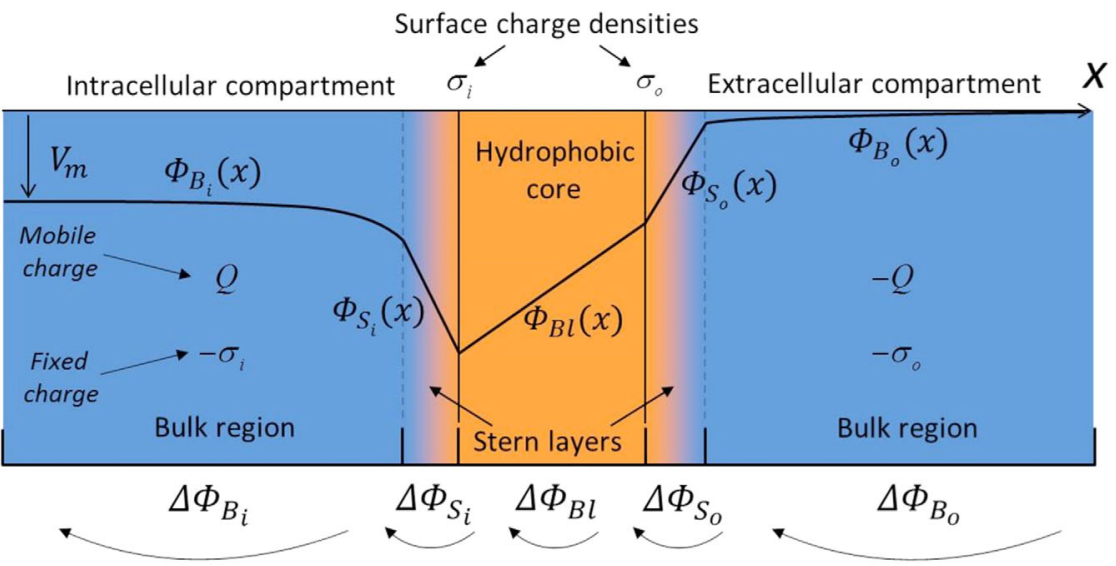

(b)

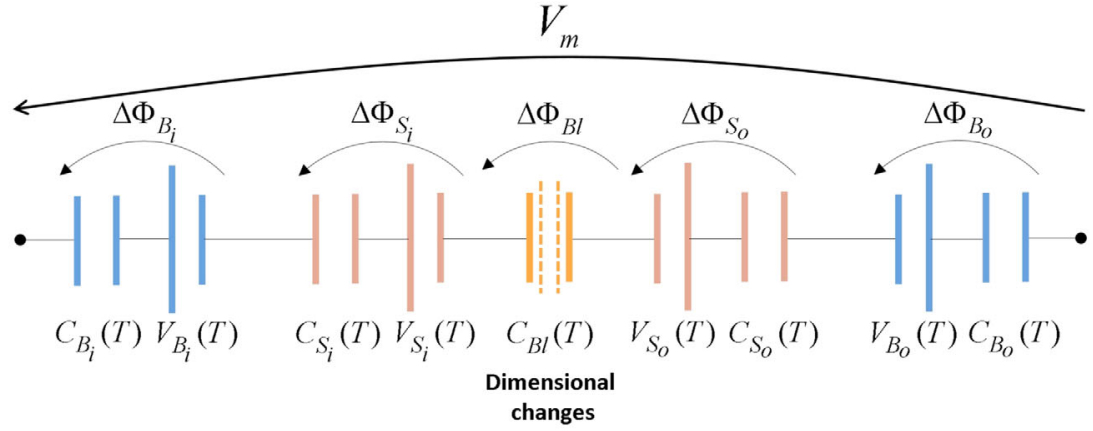

(c)

Capacitance dynamics

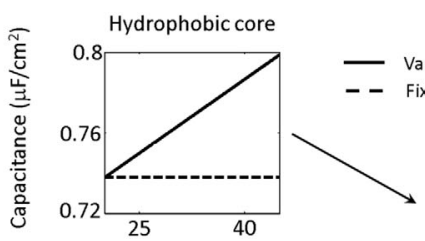

Fixed geometry

- Total capacitance outside core
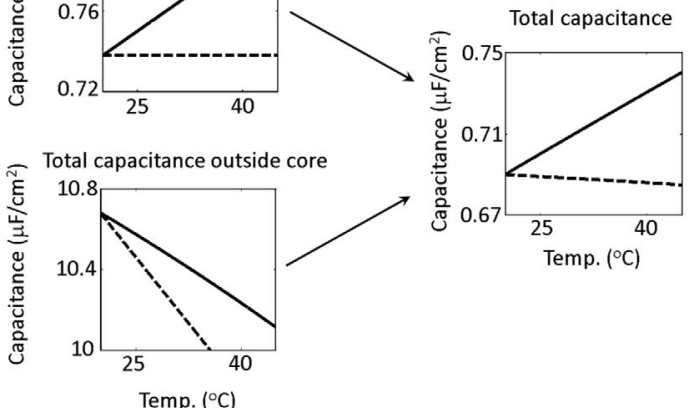

(d)

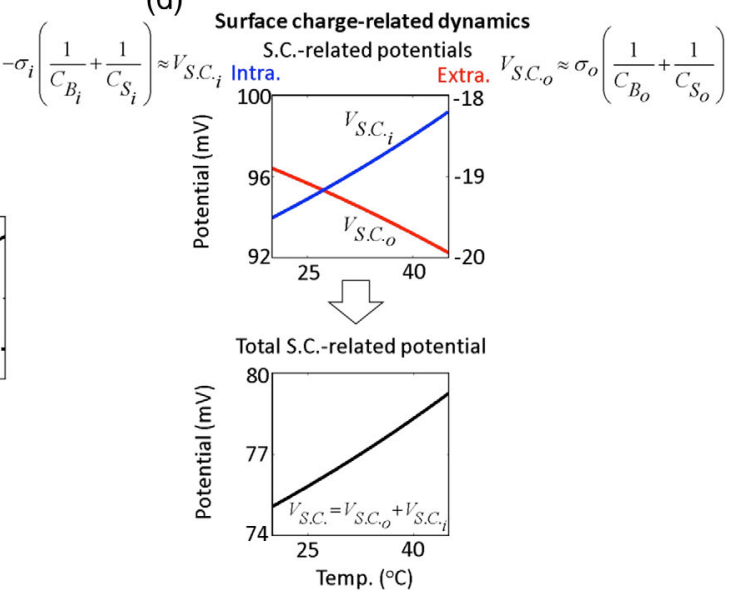

FIG. 2. Detailed (Gouy-Chapman-Stern-based) membrane biophysical model. (a) Membrane subregions: hydrophobic core, containing the phospholipid molecules' tails. The outer parts of this region contain intracellular $\left(\sigma_{i}\right)$ and extracellular $\sigma_{o}$ Unhandled Math Content: Z) surface charges. Intracellular and extracellular bulk regions containing mobile charges $(Q$ and $-Q)$ and the surface charges' fixed counterions $\left(-\sigma_{i}\right.$ and $\left.-\sigma_{o}\right)$. Two Stern layers composed of the phospholipid polar heads and the closest ions to the membrane. $\Delta \Phi_{j}$ is the potential drop on each subregion (total, $V_{m}=\sum \Delta \Phi_{j}$ ). (b) Membrane series circuit where capacitors and sources approximate each subregion $\left(\Delta \Phi_{j}=Q / C_{j}+V_{j}\right) . V_{j} \approx-\sigma_{i} / \mathrm{C}_{j}$ for $j=B_{i}$ or $S_{i}$ and $V_{j} \approx \sigma_{o} / \mathrm{C}_{j}$ for $j=B_{o}$ or $S_{o}$ is the potential resulting from respective surface charges and both $C_{j}$ and $V_{j}$ are temperature dependent. Overall, $Q=C_{m}\left(V_{m}-V_{\text {S.C. }}\right)$, where $V_{\text {S.C. }}$ is the total surface chargerelated potential. (c) Predicted thermal changes in partial and total membrane capacitance under alternative models: fixed geometry model predicts a net reduction while the varying-dimension model correctly predicts an increase [see also Fig. 1(a)]. (d) Predicted thermal response of intracellular, extracellular (upper panel), and total (bottom panel) surface charge-related potentials.

The relationships between the mobile, fixed charges, and the potentials that fall on each membrane region are determined by electrostatic Boltzmann equations in the bulk, while in the hydrophobic core and Stern layers these relations are determined through the assumption of zero density of free charges [16,38,39] (see Sec. IV). Interestingly, approximately linear relations between the mobile charge and the potentials are found in all five 
TABLE I. Variables used in the theoretical framework.

\begin{tabular}{|c|c|}
\hline Variable & Variable meaning \\
\hline$Q$ & Mobile membrane charge density \\
\hline$\sigma_{i}$ and $\sigma_{o}$ & $\begin{array}{l}\text { Inner and outer fixed membrane charge } \\
\text { density }\end{array}$ \\
\hline $\begin{array}{l}\Delta \Phi_{B l}, \Delta \Phi_{B_{i}}, \Delta \Phi_{S_{i}} \\
\quad \Delta \Phi_{B_{o}}, \Delta \Phi_{S_{o}}\end{array}$ & $\begin{array}{l}\text { Potentials across the hydrophobic region, } \\
\text { and across the internal and external bulk } \\
\text { and Stern regions, respectively }\end{array}$ \\
\hline $\begin{array}{l}C_{B l}, C_{B_{i}}, C_{S_{i}} \\
\quad C_{B_{o}}, C_{S_{o}}\end{array}$ & $\begin{array}{l}\text { Capacitances across the hydrophobic } \\
\text { region, and across the internal and } \\
\text { external bulk and Stern regions, } \\
\text { respectively }\end{array}$ \\
\hline$V_{B_{i}}, V_{S_{i}}, V_{B_{o}}, V_{S_{o}}$ & $\begin{array}{l}\text { Surface charge-related potentials in internal } \\
\text { and external bulk and Stern regions, } \\
\text { respectively }\end{array}$ \\
\hline$V_{\text {S.C. }}$ & $\begin{array}{l}\text { Total membrane surface charge-related } \\
\text { potential }\end{array}$ \\
\hline$V_{m}, I_{m}, C_{m}$ & $\begin{array}{l}\text { Membrane potential, current and } \\
\text { capacitance }\end{array}$ \\
\hline$c_{l}$ & $\begin{array}{l}\text { Concentration close to the membrane of } \\
\mathrm{Mg}^{2+} \text { or } \mathrm{Gd}^{3+}\end{array}$ \\
\hline$c_{h}$ & $\begin{array}{l}\text { Concentration close to the membrane of } \\
\mathrm{Na}^{+} \text {ions }\end{array}$ \\
\hline$z_{l}$ & Valency of $\mathrm{Mg}^{2+}$ or $\mathrm{Gd}^{3+}$ \\
\hline
\end{tabular}

membrane regions (Fig. S1 of Supplemental Material [40]), allowing the membrane to be represented as a lumped circuit of 5 temperature-dependent capacitors with surface charge-related sources in series [alternatively, as a single equivalent temperature-dependent membrane capacitor and surface charge-related source; see Fig. 2(b) and Sec. IV].

We first examine the fundamental response to temperature changes of the new membrane biophysical model and of an equivalent fixed-geometry model [16]. The fixedgeometry model predicts a paradoxical net reduction with temperature of the total membrane capacitance $\left(-0.03 \% /{ }^{\circ} \mathrm{C}\right)$ dictated by a reduction of the capacitance outside the membrane core $\left[-0.4 \% /{ }^{\circ} \mathrm{C}\right.$, Fig. 2(c); see Secs. III and IV for explanation of earlier misinterpretations $[16,26]]$. In contrast, in the new model, the membrane's hydrophobic core dimensional variations [Fig. 1(b)] contribute to a linear increase of $0.29 \pm 0.05 \% /{ }^{\circ} \mathrm{C}$ in the total membrane capacitance [Figs. 1(a) and 2(c), right-hand panel], which is dominated by this relatively small capacitor, a value that accurately matches the mean experimental capacitance change rate [Fig. 1(a)]. Importantly, although the Stern and bulk capacitances actually undergo a concurrent temperature-dependent drop [Fig. 2(c)] caused by the reduction in the surrounding dielectric constants and an elevation of the membrane's thermal or electrical forces, this effect is responsible for another surface charge-related phenomenon: an increase in the absolute values of the potentials falling on the Stern and bulk regions, contributing to an overall elevation of the total surface chargerelated potential $V_{\text {S.C. }}$ [Fig. 2(d)].

\section{Experimental validation and inference}

We next studied the model's response to a temperature transient used extensively by Shapiro et al. in their artificial membranes' experiments and model simulations [Fig. 3(a)]. The model simulations take into account the contribution of two independent thermodynamic variables, temperature and membrane voltage, and the resulting displacement current is a product of the membrane effective thermoelectric capacitance $\left(C_{m}^{T}=[(\partial Q) / \partial T]\right.$; see Ref. [27]) and the temperature time derivative (see Sec. IV E). The resulting membrane currents and potential changes were composed of similar relative contributions from the two underlying mechanisms related to capacitance and surface charge-related potential variations [Figs. 3(b) and 3(c)]. Importantly, the membrane capacitance-related current is dependent on the clamped membrane potential, while the surface charge-related current is independent [Fig. 3(b)].

Can these dynamical responses quantitatively predict the results of INS experiments in artificial bilayer membranes [16]? In simulations of phosphatidylethanolamine (PE): phosphatidylcholine (PC):phosphatidylserine (PS) and PE: PC membranes (energy $7.3 \mathrm{~mJ}$; duration $10 \mathrm{msec}$, see Secs. IV D and IV G), the resulting INS temperature transient caused a membrane capacitance increase of about $0.3 \% /{ }^{\circ} \mathrm{C}$, in agreement with the respective experimental measurements [16] [Fig. 3(a)]. The same high level of agreement was also obtained in voltage clamp simulations for simulated membrane currents and temperature elevation phase-induced membrane charge $(\Delta Q)$ in PE:PC [Figs. 3(d) and 3(e)] and PE:PC:PS [Figs. 3(f) and 3(g)] membranes. For PE:PC:PS membranes where multivalent ions were added to the pseudo "extracellular compartment" $\left(\mathrm{Mg}^{2+}\right.$ or $\mathrm{Gd}^{3+}$, as $14 \mathrm{mM} \mathrm{MgCl} 2$ or $1 \mathrm{mM} \mathrm{GdCl}$; see Sec. IV for modeling detail), the membrane currents were shifted towards more depolarizing currents due to changes in surface charge difference between the extracellular and intracellular compartments. This surface charge difference elicited a depolarizing current [shown earlier in Fig. 3(b)], which was absent when the solutions on both sides of the membrane were symmetrical [Figs. 3(f) and 3(g)].

Our final analyses examined whether the new biophysical model can also predict and explain thermostimulation results in intact living cells. First, we simulated INS stimulation experiments performed in Xenopus laevis oocytes [16] (see Table SI in Supplemental Material for simulation parameters [40]), finding a high degree of agreement between the model predictions and the experimental measurements of both currents and potentials [Figs. 4(a) and 4(b)]. Next, we performed a model-based reanalysis of our recent experiments where thermal excitation mediated by photoabsorption of $\mathrm{cw}$ laser pulses with varying durations was used to excite cells in rat cortical cultures [18]. Farah et al. [18] observed that stimulation thresholds are well predicted if putative depolarizing displacement currents proportional 
(a)

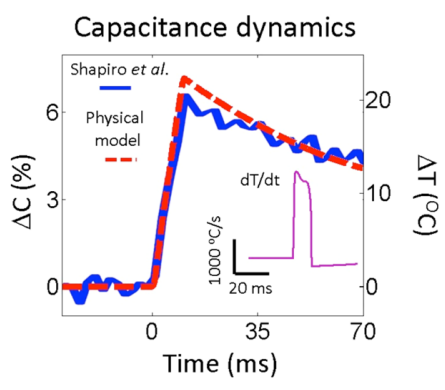

(b) $I_{m}=\frac{I_{C_{m}}}{\frac{d C_{m}}{d t} \cdot\left[V_{m}-V_{S . C .}\right]-C_{m} \cdot \frac{I_{S . C .}}{d V_{S . C .}}}$

(c)
$V_{m}=\frac{\frac{V_{C_{m}}}{Q}}{C_{m}}+V_{S . C .}$
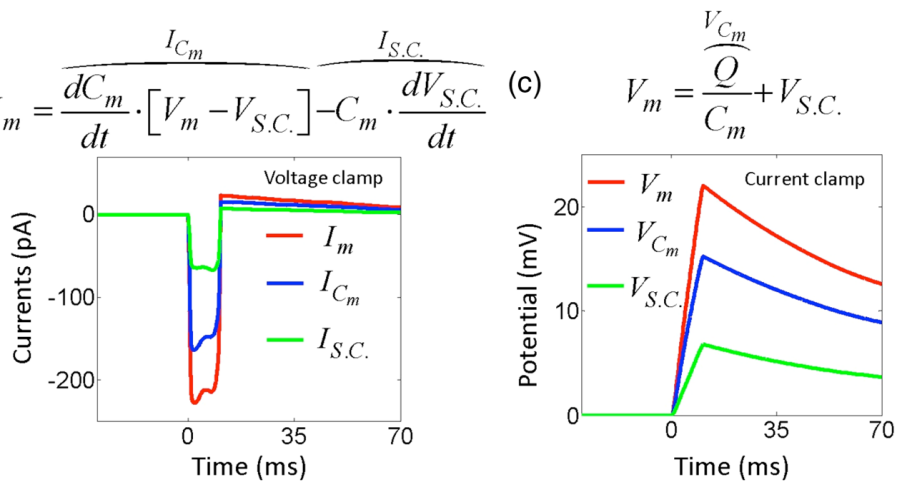

(d)

PE:PC membranes

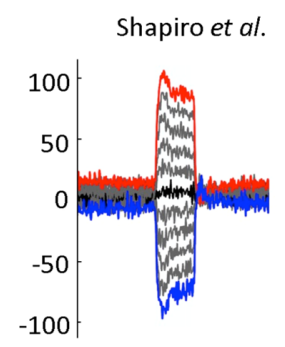

(f)

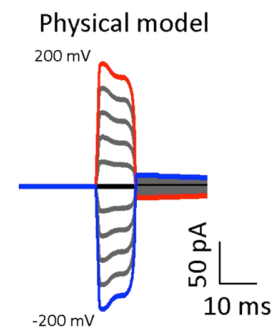

(e)

PE:PC membranes

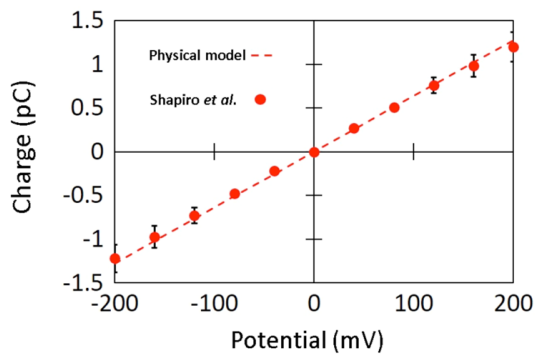

(g)

PE:PC:PS membranes
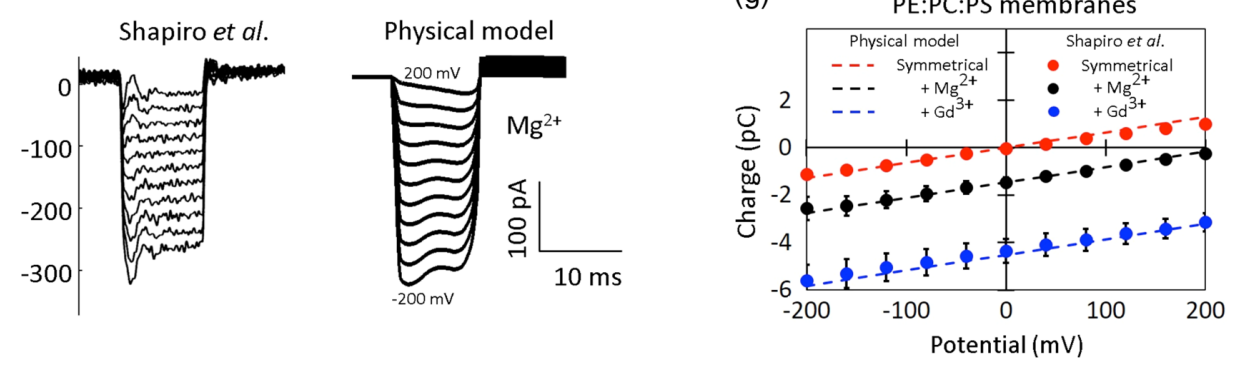

FIG. 3. Empirical results versus model-based predictions for artificial bilayer membrane INS experiments. (a) Predicted versus measured capacitance dynamics for $10 \mathrm{~ms}$ pulses. The capacitance change is proportional to the temperature transient (inset: temperature time derivative). (b),(c) Underlying membrane currents and potentials in response to the temperature transient in (a). The overall response is determined by the additive contribution of the membrane capacitance and surface charge-related potential thermal responses. (d)-(g) Predicted versus measured thermal membrane currents (d),(f) and induced charge (e),(g) for PE:PC membranes and for PE:PC: PS membranes. Addition of $14 \mathrm{mM} \mathrm{MgCl}_{2}$ (f), (g) or $1 \mathrm{mM} \mathrm{GdCl}_{3}$ (g) to the "extracellular" compartment shifts towards more depolarizing currents through membrane absorption of excess ions changing the extracellular surface charge density. The simulations use the experimental parameters of Shapiro et al. [16] (Table SI of Supplemental Material [40]). Error bars are \pm s.e.m.

to the temperature's time derivative, $I_{L}=-\alpha[(d T) / d t]$ $\left[\alpha=2.53 \times 10^{-5} \mathrm{C} /{ }^{\circ} \mathrm{C} / \mathrm{m}^{2}\right.$, Fig. $4(\mathrm{~d})$, based on Fig. 5(a) of Ref. [18]], are inserted into model layer $V$ cortical neurons. Reexamining in terms of the new biophysical model, $\alpha$ is almost entirely dependent on the temperature derivative of the membrane capacitance multiplied by the surface charge-related potential, allowing us to estimate the surface charge difference, $\Delta \sigma=\sigma_{i}-\sigma_{o}$, as $-0.107 \mathrm{C} / \mathrm{m}^{2}$ plus maximal deviation $\approx 5 \%$ (note that $\alpha$ is identified as the neural membrane's thermoelectric capacitance; see Secs. IVE and IV F for details). This result is consistent with the surface charge density range of $0.002-0.37 \mathrm{C} / \mathrm{m}^{2}$ that was measured in neural cells [41].

\section{DISCUSSION}

This study explored the effects of temperature changes on membrane capacitance and its associated currents in a joint attempt to clarify the experimental results of a key recent study [16] and to pave the way towards predictive modeling of INS [2-15] and other thermal neurostimulation techniques [18-20], which could potentially facilitate 
the development of more advanced and multimodal methods for neural circuit control. Another key motivation to pursue this problem came from our noting the very similar temperature-related capacitance rates of change observed in very different model systems [Fig. 1(a)] suggesting that this value is putatively universal.

Although the pioneering study by Shapiro et al. [16] also sought to unravel their results' underlying biophysical mechanism, upon further scrutiny we found that their fixed-geometry GCS peripheral capacitances (bulk and Stern) decrease with temperature $[28,29]$. Our new model leads to a diametrically different qualitative story, where the underlying phenomenon is now instead attributed to the following two completely novel mechanisms.

(1) Thermal membranal dimensional changes inferred from small-angle $x$-ray and neutron scattering measurements, and molecular dynamics simulations [33]. These lead to an increase in the overall capacitance and to capacitive displacement currents $\left[I_{m}=d C_{m} / d t\left(V_{m}-V_{\text {S.C. }}\right)\right.$, Figs. 3(a) and 3(b)]. These dimensional changes have been observed in a range of different model membranes, including POPC, SOPC, DPhyPC, and more [30-33], and are putatively attributed to entropically driven shortening of the phospholipids' tails [Fig. 1(b)], a process termed trans-gauche isomerization [31,33]. The predicted associated capacitance rates are relatively insensitive to the specific choice of a membrane lipid composition $\left(0.33 \pm 0.05 \% /{ }^{\circ} \mathrm{C}\right.$ for SOPC and $0.32 \pm 0.06 \% /{ }^{\circ} \mathrm{C}$ for DPhyPC), mixture ratios (e.g., as seen in our simulations of the different mixtures in Ref. [16]), and baseline temperature $[31,33]$.

(2) Thermally mediated displacement currents $\left(I_{m}=-C_{m} d V_{\text {S.C. }} / d t\right)$ resulting from an increase of the surface charge-related potential [Fig. 3(b)].

This dual mechanism found by separately considering the temperature-dependent behavior in each membrane subregion explains the experimental shift of membrane current when multivalent ions $\left(\mathrm{Mg}^{2+}\right.$ and $\left.\mathrm{Gd}^{3+}\right)$ that affect the surface charge [42] were added to one side of the membranes' solutions [16], while almost no effect was observed on membrane capacitance variations [slope in Figs. 3(e) and 3(g)]. Added together, the net thermal displacement current equals the product of the thermoelectric capacitance and the temperature's time derivative (see Sec. IVE and Ref. [27]), specifically allowing for the PAINTS experiment to estimate a surface charge difference that was found to be in accord with empirical values for neural cells [41].

The theoretical analysis of the META model was found to both qualitatively and quantitatively explain the results of artificial membrane, oocyte, and cortical culture experiments, using parameters which were taken "as is" from the literature, whenever attainable (Figs. 3 and 4). However, it should be noted that the model contains key simplifying assumptions and that other phenomena could also potentially contribute during the thermal stimulation process.

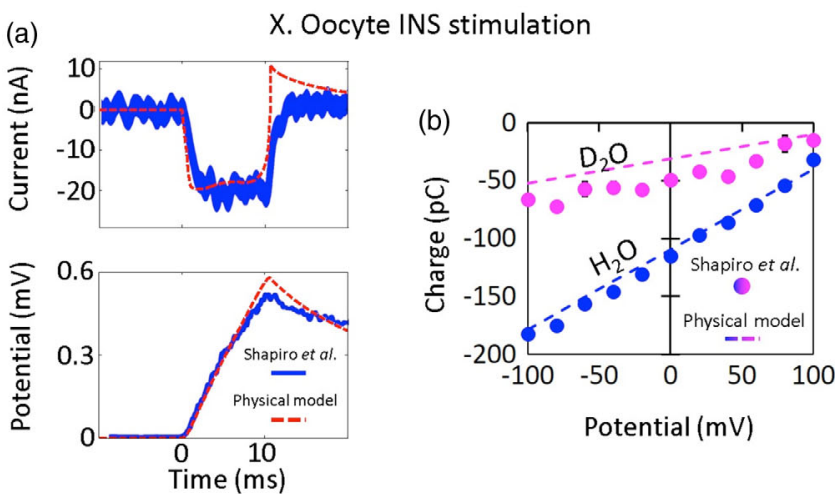

Photoabsorber-induced neural-thermal stimulation

(c) Suprathreshold excitation
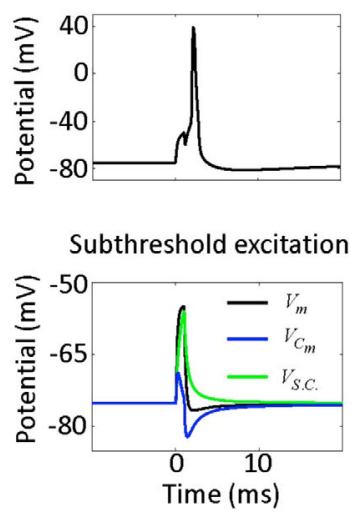

(d) Farah et al. results

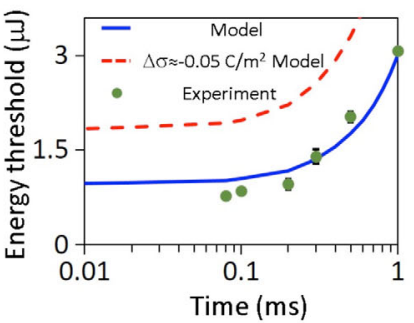

FIG. 4. Empirical cellular neurostimulation results versus modelbased predictions. (a),(b) Predicted versus measured membrane currents, potentials, and induced charge, for $10 \mathrm{~ms}, 7.3 \mathrm{~mJ}$ INS stimulation of Xenopus oocyte in $\mathrm{H}_{2} \mathrm{O}$ - and $\mathrm{D}_{2} \mathrm{O}$-based extracellular media; illumination of $5 \%$ of the surface area assumed [16]. (c) Predicted somatic membrane potentials in a cortical pyramidal neuron stimulated by threshold and $90 \%$ subthreshold simulated $1 \mathrm{~ms}$ PAINTS transients. The membrane potential $\left(V_{m}\right)$ thermal response is dictated by surface charge- and capacitance-related potentials ( $V_{\text {S.C. }}$ and $V_{C m}$, respectively), where $V_{C m}$ is also affected by the membrane's charge dynamics. (d) Predicted versus empirical thresholds [Fig. 5(a) in Ref. [18]]. The constant $\alpha$ determined from the best fit was used to calculate the surface charge difference in rat cortical neurons (solid blue line, $\Delta \sigma=\sigma_{i}-\sigma_{o}=-0.107 \mathrm{C} / \mathrm{m}^{2}$ ); predictions for an alternative model $\left(\Delta \sigma=-0.05 \mathrm{C} / \mathrm{m}^{2}\right.$, dashed red line) are also shown for comparison. $\sigma_{i} \approx-0.12 \mathrm{C} / \mathrm{m}^{2}$ and $\sigma_{o} \approx-0.013 \mathrm{C} / \mathrm{m}^{2}$ for $V_{C m}$ and $\mathrm{V}_{\text {S.C. }}$ the calculation in (c). Error bars are \pm s.e.

First, the membrane's hydrophobic core's dielectric constant was considered as temperature independent. This simplification is largely based on the only relatively minor thermal effects on dielectric constants observed in experiments performed on different types of fatty acids $\left(-0.019 \pm 0.008 \% /{ }^{\circ} \mathrm{C}, \pm\right.$ s.e.m., over 1 order of magnitude lower than the capacitance thermal rate considered; see Ref. [34]). A concern that prevented including even this minor effect into the model is that these measurements are sensitive to, and thus may potentially be confounded by, 
complex experimental interactions with changes of a similar magnitude, which are caused by variations in the effective dielectric constant of the ionic solution around the membrane (as seen in our model results). Second, we used values which were measured in an ion-free medium to model membranes immersed in physiological ion solutions. We anticipate that the likely impact of this simplification is also relatively minor. Experimentally, rather negligible effects on the membranes' structural parameters were observed when the concentration of ions like $\mathrm{Na}^{+}$, $\mathrm{Cl}^{-}$, and $\mathrm{Ca}^{2+}$ was varied within their physiological ranges [43-45]. In addition, no effect on the thermal capacitance rate was noted in the experiments of Shapiro et al. when the multivalent ions $\mathrm{Mg}^{2+}$ and $\mathrm{Gd}^{3+}$ were added to the solution, even at concentrations that are higher than the physiological range [see the parallel slopes in Figs. 3(g)]. Finally, preliminary results we obtained from molecular dynamics simulations of membranes in a $150 \mathrm{mM} \mathrm{NaCl}$ solution also show a relatively minor effect on the predicted thermal capacitance rate, not exceeding its $\sim 20 \%$ expected error range (data not shown). Taken together with the excellent and detailed agreement of the new model with a wide array of experimental results leads us to believe that it is safe to assume that there is currently no clear indication of a need to necessarily complicate the model.

Nevertheless, attempts to develop a comprehensive understanding of thermal neuroexcitation phenomena will clearly need to explore possible interactions between META and auxiliary effects. Phospholipid phase transitions (putatively not present in the quasilinear regime explored in Ref. [16] and here) can have a profound influence on membrane structure and thus on membrane capacitance. The classical observations by Palti and Adelman [23] observed steep orders of magnitude increases in squid axonal membrane thermal capacitance rates between $3{ }^{\circ} \mathrm{C}-20^{\circ} \mathrm{C}$ (likely attributed to gel to liquid-crystalline phase transition [46]) and above $42^{\circ} \mathrm{C}$ (likely a transition to hexagonal phase [46]). Interestingly, these results anecdotally suggest that thermal stimulation methods will likely be much more effective in mammalian in vivo experiments where temperature elevations can practically reach a phase transition. Also, the identified mechanoelectrical effects are dominant in the short time scales considered here, but in conjunction with parallel thermal effects like changes in the $Q_{10}$ factor [47], probably play a significant role under a wide array of thermal modulation scenarios [48]. Related insights may also help guide our understanding of other emerging neurophysical modalities like magnetogenetic stimulation (whose biophysics is still poorly understood [49,50]). For example, we note that membrane mechanoelectrical effects involving dimensional changes were suggested in other contexts involving changes in intramembranal forces, including action potential-related intramembrane thickness variations [51-53] and ultrasoundinduced formation of intramembrane cavities (or "bilayer sonophores" [54]). The neuronal intramembrane cavitation excitation theoretical framework putatively explains ultrasonic neuromodulation phenomena (suppression and excitation [55]) and predicts the results of a significant number of related experimental studies [56,57].

\section{METHODS}

\section{A. Constitutive electrostatic laws and equations}

The relation between the mobile $(Q)$ and fixed $(\sigma)$ membrane charge densities and the potentials $(\Delta \Phi)$ that fall on the bulk regions [Fig. 2(a)] is obtained through electrostatic Boltzmann equations [16,38] (see Table I for a list of model variables):

$$
\begin{array}{ll}
\text { intracellular bulk: } & \left(\sigma_{i}-Q\right)^{2}=2 \varepsilon_{T_{\text {buk }_{i}}} R T \sum_{j=1}^{n} c_{j}(-\infty)\left[\exp \left(\frac{z_{j} F}{R T} \Delta \Phi_{B_{i}}\right)-1\right], \\
\text { extracellular bulk: } & \left(\sigma_{o}+Q\right)^{2}=2 \varepsilon_{T_{\text {bulk }_{o}}} R T \sum_{j=1}^{n} c_{j}(\infty)\left[\exp \left(-\frac{z_{j} F}{R T} \Delta \Phi_{B_{o}}\right)-1\right],
\end{array}
$$

where $\varepsilon_{T_{\text {bulk }_{i}}}$ and $\varepsilon_{T_{\text {bulko }_{o}}}$ are the temperature-dependent intracellular and extracellular dielectric constants, respectively; $R$ and $F$ are the ideal gas and Faraday constants, respectively; $T$ is the membrane's surrounding temperature; $c_{j}(-\infty)$ and $c_{j}(\infty)$ are the intracellular and extracellular ion concentrations far from the membrane; and $z_{j}$ is ion valence.

The relation between membrane charge and the potentials that fall on the Stern regions can be obtained through the following mathematical formulations [16,39], which take into account the respective dielectric constants of the subdivisions of the Stern layer (polar heads and ionic region):

$$
\begin{array}{ll}
\text { intracellular Stern region: } & \Delta \Phi_{S_{i}}=\left(Q-\sigma_{i}\right)\left[\frac{\delta_{\text {lipid }}}{\varepsilon_{H}}+\frac{1}{\varepsilon_{R_{i}}} \frac{\sum_{j=1}^{n} r_{j} c_{j}(-\infty) \exp \left(\frac{z_{j} F}{R T} \Delta \Phi_{B_{i}}\right)}{\sum_{j=1}^{n} c_{j}(-\infty) \exp \left(\frac{z_{j} F}{R T} \Delta \Phi_{B_{i}}\right)}\right], \\
\text { extracellular Stern region: } & \Delta \Phi_{S_{o}}=\left(Q+\sigma_{o}\right)\left[\frac{\delta_{\text {lipid }}}{\varepsilon_{H}}+\frac{1}{\varepsilon_{R_{o}}} \frac{\sum_{j=1}^{n} r_{j} c_{j}(\infty) \exp \left(-\frac{z_{j} F}{R T} \Delta \Phi_{B_{o}}\right)}{\sum_{j=1}^{n} c_{j}(\infty) \exp \left(-\frac{z_{j} F}{R T} \Delta \Phi_{B_{o}}\right)}\right],
\end{array}
$$


where $\Delta \Phi_{S_{i}}$ and $\Delta \Phi_{S_{o}}$ are the intracellular and extracellular potentials, respectively, that fall on the Stern layers [Fig. 2(a)]; $r_{j}$ is the ionic effective radius and $\varepsilon_{R_{i}}, \varepsilon_{R_{o}}$, and $\varepsilon_{H}$ are the temperature-dependent dielectric constants in the intracellular and extracellular ionic regions and in the lipid polar heads, respectively. We took $\varepsilon_{R}=\varepsilon_{T_{\text {bulk }}}$ (which is plausible for surface charge density $\leq 0.2 \mathrm{C} / \mathrm{m}^{2}$ [58]the maximal charge density for fluid phase membranes [42]) and $\varepsilon_{H}=\frac{25.6}{80} \varepsilon_{T_{\text {bulk }}}$ [59].

The relation between the membrane's mobile charge and the potential that falls on the hydrophobic core region is linear, under the assumption of zero density of free charges [38]:

$$
Q=C_{B l} \Delta \Phi_{B l},
$$

where $C_{B l}$ and $\Delta \Phi_{B l}$ are the hydrophobic region capacitance and potential. Interestingly, Eqs. (1) and (2) can be seen to yield additional approximately linear relations for the four external membrane regions (see Fig. S1 of Supplemental Material [40]), wherein

$$
Q=C_{k}\left[\Delta \Phi_{k}-V_{k}\right]
$$

$\Delta \Phi_{k}$ is the potential that falls on each membrane region and $C_{k}$ and $V_{k}$ are their respective capacitances and surface charge-related potentials $\left(k \in B_{i}, S_{i}, B l, S_{o}\right.$, or $B_{o} ; V_{B_{i}} \approx\left[\left(-\sigma_{i}\right) / C_{B_{i}}\right], \quad V_{S_{i}} \approx\left[\left(-\sigma_{i}\right) / C_{S_{i}}\right], \quad V_{B l}=0$, $V_{B_{o}} \approx\left(\sigma_{o} / C_{B_{o}}\right)$, and $\left.V_{S_{o}} \approx\left(\sigma_{o} / C_{S_{o}}\right)\right)$.

Following the relations shown above, the membrane can be represented as a lumped circuit of 5 capacitors with surface charge-related sources in series, where $C_{k}(T)$ and $V_{k}(T)$ are temperature-dependent parameters, which allow us to obtain a simple mathematical linear formula for membrane charge and potential $\left(V_{m}\right)$ [Fig. 2(b)]:

$$
Q\left(V_{m}, T\right)=C_{m}(T)\left[V_{m}-V_{\text {S.C. }}(T)\right],
$$

where $C_{m}(T)=\left\{\sum_{k}\left[1 / C_{k}(T)\right]^{-1}\right\}$ is the total membrane capacitance and $V_{\text {S.C. }}(T)=\sum_{k} V_{k}(T)$. Throughout the study $C_{m}\left(20^{\circ} \mathrm{C}\right)$ was obtained from measurements, and used together with the four capacitance values from Eqs. (1) and (2) to calculate the unknown capacitance $C_{B l}$ [except in the theoretical analysis of the temperature effects shown in
Figs. 1 and 2, where we used a reference value $C_{B l}\left(20^{\circ} \mathrm{C}\right)$ calculated in the model of Shapiro et al.].

\section{B. Membrane mobile charge notation}

Genet et al. [38] defined the extracellular mobile charge as $Q(Q>0)$ and the intracellular charge as $-Q$. These definitions are opposite of the standard convention, and their naive application in simulating currents will lead to $I \triangleq[(d Q) / d t]=-I_{m}$, where $I_{m}$ is a conventional membrane current defined positive for a current flowing from the intracellular to the extracellular domain. It appears that several authors may have made this error [16,26]. In Sec. II, the fixed geometry model avoids this notational error [Fig. 2(c)].

\section{Dimensional variations}

A major conceptual difference between ours and earlier models is the incorporation here of temperature-dependent variations in the membrane's hydrophobic region dimensions. Estimates of the temperature dependence of the hydrophobic core dimensions were based on published measurements that used x-ray and neutron small-angle scattering experiments and molecular dynamics simulations [31,33], selecting the characteristics of 1-palmitoyl-2oleoyl-snglycero-3-phosphatidylcholine (POPC; see Table II for its dimensional change characteristics), which in the relevant temperature range of $20^{\circ} \mathrm{C}<\mathrm{T}<45^{\circ} \mathrm{C}$ is in its liquid-crystalline state. POPC was selected because it relatively closely mimics the mammalian phospholipid composition [60] and is representative of the thermal response of various liquid-crystalline lipid membranes [30-33], including lipid mixtures as used by Shapiro et al. [16] (Fig. 1). The hydrophobic core thermal response also modifies Eqs. (1) and (2) by noting that the charge values correspond to areal charge densities.

\section{Membrane ions absorption}

In artificial membrane simulations under the experimental conditions of Shapiro et al. [16] (symmetrical membranes $-1: 1: 1 \mathrm{PE}: \mathrm{PC}: \mathrm{PS}$ and $1: 1 \mathrm{PE}: \mathrm{PC}$, wherein the PS is a negatively charged phospholipid molecule, while the PE and PC are nearly uncharged [61]), surface charge change due to ions absorption were modeled through the Langmuir isotherm [42],

TABLE II. POPC membranes' thermal dimensional change parameters used in the theoretical framework (see Refs. [31,33]).

\begin{tabular}{lccc}
\hline \hline Structural parameter & Value $\left(20^{\circ} \mathrm{C}\right)$ & Thermal rate change $\left(\right.$ per $\left.{ }^{\circ} \mathrm{C}\right)$ & Relative thermal rate $\left[\% /{ }^{\circ} \mathrm{C}\right]$ \\
\hline $\boldsymbol{\delta}[\AA]$ & $29.2 \pm 0.6$ & $-0.031 \pm 0.010$ & $-0.11 \pm 0.03$ \\
$\boldsymbol{A}$ (membrane area per lipid) $\left[\AA^{2}\right]$ & $62.7 \pm 1.3$ & $0.14 \pm 0.02$ & $0.22 \pm 0.03$ \\
$\boldsymbol{V}$ (hydrophobic zone volume) $\left[\AA^{3}\right]$ & $1831 \pm 53$ & $2.14 \pm 0.86$ & $0.12 \pm 0.05$ \\
\hline \hline
\end{tabular}




$$
\sigma=\frac{P S}{f_{P S} S\left[\frac{\left(z_{l}-1\right) K_{l-P S} c_{l}-1}{1+K_{h} c_{h}+K_{l-P S} c_{l}}\right]}+\frac{P E \text { and } P C}{f_{P E \text { and } P C} S\left[\frac{z_{l} K_{l-P E / P C} c_{l}}{1+K_{l-P E / P C} c_{l}}\right]}
$$

where $S$ is the maximal charge density of the ions' potential membrane binding sites - equals to $0.2 \mathrm{C} / \mathrm{m}^{2}$ that corresponds to $0.6 \mathrm{~nm}^{2}$ per lipid molecule for membranes in fluid phase condition [31,42]; $f_{\mathrm{PS}}$ and $f_{\mathrm{PE}}$ and $\mathrm{PC}$ are the molecular fraction of PS and PE/PC; $z_{l}, c_{l}$, and $K_{l-\mathrm{PS}}$ are the valency, concentration close to the membrane, and absorption factor to PS of the multivalent $\mathrm{Mg}^{2+}$ or $\mathrm{Gd}^{3+}$ ions [16], respectively; $K_{l-\mathrm{PE} / \mathrm{PC}}$ is the multivalent ions' absorption factor to PE and PC $[42,62]$; and $c_{h}$ and $K_{h}$ are the concentration close to the membrane and absorption factor to PS of the $\mathrm{Na}^{+}$ions [63] (see Table SI in Supplemental Material [40] for parameter values).

\section{E. Current and potential calculations}

Solving the GCS-based model allowed us to calculate the expected temperature-induced membrane currents in a voltage clamp mode:

$$
\begin{aligned}
I_{m} & =\frac{d Q}{d t}=\frac{d\left(C_{m}(T) \cdot\left[V_{m}-V_{\text {S.C. }}(T)\right]\right)}{d t} \\
& =C_{m} \cdot \frac{\int_{\text {Voltage Clamp }}}{\frac{d V / m}{d t}+\left(\frac{d C_{m}}{d T} \cdot\left[V_{m}-V_{\text {S.C. }}\right]-C_{m} \cdot \frac{\left.d V_{\text {S.C. }}\right)}{d T}\right)} \frac{d T}{d t}
\end{aligned}
$$

where $(d T) / d t$ is the temperature time derivative, $\left[\left(d C_{m}\right) / d T\right][(d T) / d t]\left[V_{m}-V_{\text {S.C. }}\right]$ is the membrane capacitive current that arises due to changes in the total membrane capacitance, $-C_{m}\left[\left(d V_{\text {S.C. }}\right) / d T\right]$ $[(d T) / d t]$ is the surface charge-related current, and $C_{m}^{T}$ is the thermoelectric capacitance according to $I_{m}=d Q / d t=\partial \overparen{C_{m}} / \partial V_{m} d V_{m} / d t+\partial \overparen{C_{m}^{T} / \partial T} d T / d t$ presented in Ref. [27]. The membrane potential in a current clamp mode is expressed as

$$
V_{m}=\frac{Q}{C_{m}(T)}+V_{\text {S.C. }}(T)
$$

\section{F. Estimation of surface charge difference in neurons}

To estimate the intracellular and extracellular surface charge difference in neurons, we used our recent discovery that temperature transients excite neurons through formation of membrane currents that are proportional to the temperature time derivative, $I_{L}=-\alpha[(d T) / d t]$, where $\alpha$ is a positive constant [18], which according to the GCS-based model actually equals to the effective thermoelectric capacitance:

$$
I_{L}=-\frac{\alpha}{\left(\frac{d\left(C_{m} \cdot V_{S . C}\right)}{d T}-V_{m} \frac{d C_{m}}{d T}\right)} \cdot \frac{d T}{d t}
$$

Although $V_{m} * d C_{m} / d T$ is dependent on the membrane potential, its maximal variation of several percent $(4 \%-$ $7.5 \%$ ) prior to the action potential formation negligibly affects the $\alpha$ value; the main weight of the linear temperature-dependent parameter, $C_{m} V_{\text {S.C. }}\left(R^{2} \geq 0.999,20^{\circ} \mathrm{C} \leq\right.$ $T \leq 50^{\circ} \mathrm{C}$ ) explains the emergence of $\alpha$ as a constant in the temperature rate model of Farah et al. [18]. Since the membrane surface charge densities determine the $V_{\text {S.C. }}$ (see above), it is possible to extract the neural surface charge density difference by knowing the $\alpha$. For accuracy, the calculations account for the variation in $V_{m} * d C_{m} / d T$ by taking the average of the membrane potential prior to the action potential formation.

\section{G. Model simulation details}

The model simulations were conducted in MATLAB, when the solutions were divided into several steps. (1) Subregion parameters were determined explicitly in temperature steps of $0.03{ }^{\circ} \mathrm{C}\left(20{ }^{\circ} \mathrm{C}-50{ }^{\circ} \mathrm{C}\right): C_{B l}$ was extracted from the POPC thermal response [Eq. (3)], while the $C_{k}$ and $V_{k}$ in Stern and bulk regions [Eq. (4)] were determined from linear regression of the potential-charge relations in Eqs. (1) and (2) (see Fig. S1 of Supplemental Material [40]); these relations allowed formulating a simple linear membrane capacitor formula [Eq. (5)]. (2) Although $C_{m}$ and $V_{\text {S.C. }}$ demonstrate quite linear temperature dependence $\left(R^{2} \geq 0.999\right.$ and $R^{2} \geq 0.998$, respectively, $20^{\circ} \mathrm{C}-50^{\circ} \mathrm{C}$ ), quadratic curve fittings were implemented for obtaining accurate temperature-dependent analytic formulas. (3) Determination of $C_{m}$ and $V_{\text {S.C. }}$ formulas led to simulations of temperature transient-related membrane currents and potentials [Eqs. (7) and (8)].

IR-induced temperature dynamics was extracted indirectly from the simulations of Shapiro et al. [16] (laser energy $-7.3 \mathrm{~mJ}$, duration $-10 \mathrm{~ms}$ ), wherein currents are proportional to temperature time derivative [Fig. 4(c) of their study]:

$$
\begin{aligned}
\frac{d T}{d t} & =\frac{\max \left(\Delta T_{\text {Shapiro }}\right)}{\max \left(\int_{0}^{t} I_{\text {Shapiro }}(\tau) d \tau\right)} I_{\text {Shapiro }}(t), \\
T(t) & =\int_{0}^{t} \frac{d T}{d \tau} d \tau
\end{aligned}
$$

when $\max \left(\Delta T_{\text {Shapiro }}\right)=22.2^{\circ} \mathrm{C}$ is the maximal temperature associated with laser energy $-7.3 \mathrm{~mJ}$ and duration $-10 \mathrm{~ms}$. 
Finally, the average artificial membrane capacitance in the experiments of Shapiro et al. at a reference temperature of $20^{\circ} \mathrm{C}$ was determined from charge-potential slopes [Figs. 3(c) and 4(f) of their study, obtained by linear regression] and maximal capacitance change percentage [Fig. 3(e) of their study] for energy $-7.3 \mathrm{~mJ}$ and duration $-10 \mathrm{~ms}$; the slopes represent temperature-induced maximal capacitance change [seen from integration of Eq. (7)]. The reference artificial membrane capacitance obtained from this analysis is $89 \pm 5.5 \mathrm{pF}$ ( \pm s.e.m.).

\section{H. Statistical analysis}

The reported errors in text are \pm chi-squares distributionrelated uncertainty [31], unless stated otherwise.

\section{ACKNOWLEDGMENTS}

This work was supported by an ERC COG No. 648927 grant, the Russell Berrie Nanotechnology Institute, the Israel Science Foundation Grant No. 1725/13, and by NYU Langone Health. We thank Shimon Marom, Dmitry Rinberg, Sarah Shaykevich, and Shelly Tzlil for helpful comments on the manuscript, and Uri Raviv, Jeff Klauda, and Norbert Kućerka for helpful discussions on models and measurements of joint thermal-ionic effects on membrane dimensions.

M. P. and S. S. designed the research. M. P. performed all model simulations and analyzed the data with S. S. E. S. performed auxiliary molecular dynamics simulations and contributed to Fig. 1(b). S. S. and E. K. supervised the project and prepared the manuscript with $\mathrm{M}$. P.

[1] O. Yizhar, L. E. Fenno, T. J. Davidson, M. Mogri, and K. Deisseroth, Optogenetics in Neural Systems, Neuron 71, 9 (2011).

[2] C.-P. Richter, A. I. Matic, J. D. Wells, E. D. Jansen, and J. T. Walsh, Neural Stimulation with Optical Radiation, Laser Photonics Rev. 5, 68 (2011).

[3] J. Wells, C. Kao, K. Mariappan, J. Albea, E. D. Jansen, P. Konrad, and A. Mahadevan-Jansen, Optical Stimulation of Neural Tissue In Vivo, Opt. Lett. 30, 504 (2005).

[4] J. Wells, P. Konrad, C. Kao, E. D. Jansen, and A. MahadevanJansen, Pulsed Laser versus Electrical Energy for Peripheral Nerve Stimulation, J. Neurosci. Methods 163, 326 (2007).

[5] A. D. Izzo, C.-P. Richter, E. D. Jansen, and J. T. Walsh, Laser Stimulation of the Auditory Nerve, Lasers Surg. Med. 38, 745 (2006).

[6] I. U. Teudt, A. E. Nevel, A. D. Izzo, J. T. Walsh, and C.-P. Richter, Optical Stimulation of the Facial Nerve: A New Monitoring Technique?, Laryngoscope 117, 1641 (2007).

[7] A. D. Izzo, J. T. Walsh, H. Ralph, J. Webb, M. Bendett, J. Wells, and C.-P. Richter, Laser Stimulation of Auditory Neurons: Effect of Shorter Pulse Duration and Penetration Depth, Biophys. J. 94, 3159 (2008).
[8] C.-P. Richter, S. M. Rajguru, A. I. Matic, E. L. Moreno, A. J. Fishman, A. M. Robinson, E. Suh, and J. T. Walsh, Spread of Cochlear Excitation during Stimulation with Pulsed Infrared Radiation: Inferior Colliculus Measurements, J. Neural Eng. 8, 056006 (2011).

[9] S. M. Rajguru, C.-P. Richter, A. I. Matic, G. R. Holstein, S. M. Highstein, G. M. Dittami, and R. D. Rabbitt, Infrared Photostimulation of the Crista Ampullaris, J. Physiol. 589, 1283 (2011).

[10] J. M. Bec, E. S. Albert, I. Marc, G. Desmadryl, C. Travo, A. Muller, C. Chabbert, F. Bardin, and M. Dumas, Characteristics of Laser Stimulation by Near Infrared Pulses of Retinal and Vestibular Primary Neurons, Lasers Surg. Med. 44, 736 (2012).

[11] J. M. Cayce, R. M. Friedman, E. D. Jansen, A. MahavadenJansen, and A. W. Roe, Pulsed Infrared Light Alters Neural Activity in Rat Somatosensory Cortex In Vivo, NeuroImage 57, 155 (2011).

[12] J. M. Cayce, R. M. Friedman, G. Chen, E. D. Jansen, A. Mahadevan-Jansen, and A. W. Roe, Infrared Neural Stimulation of Primary Visual Cortex in Non-Human Primates, NeuroImage 84, 181 (2014).

[13] M. W. Jenkins, A. R. Duke, S. Gu, H. J. Chiel, H. Fujioka, M. Watanabe, E. D. Jansen, and A. M. Rollins, Optical Pacing of the Embryonic Heart, Nat. Photonics 4, 623 (2010).

[14] G. M. Dittami, S. M. Rajguru, R. A. Lasher, R. W. Hitchcock, and R. D. Rabbitt, Intracellular Calcium Transients Evoked by Pulsed Infrared Radiation in Neonatal Cardiomyocytes, J. Physiol. 589, 1295 (2011).

[15] J. Wells, C. Kao, P. Konrad, T. Milner, J. Kim, A. Mahadevan-Jansen, and E. D. Jansen, Biophysical Mechanisms of Transient Optical Stimulation of Peripheral Nerve, Biophys. J. 93, 2567 (2007).

[16] M. G. Shapiro, K. Homma, S. Villarreal, C.-P. Richter, and F. Bezanilla, Infrared Light Excites Cells by Changing Their Electrical Capacitance, Nat. Commun. 3, 736 (2012).

[17] N. Farah, I. Brosh, C. R. Butson, and S. Shoham, PhotoThermal Neural Excitation by Extrinsic and Intrinsic Absorbers: A Temperature-Rate Model, arXiv:1201.4617.

[18] N. Farah, A. Zoubi, S. Matar, L. Golan, A. Marom, C. R. Butson, I. Brosh, and S. Shoham, Holographically Patterned Activation Using Photo-Absorber Induced NeuralThermal Stimulation, J. Neural Eng. 10, 056004 (2013).

[19] J. L. Carvalho-de-Souza, J. S. Treger, B. Dang, S. B. H. Kent, D. R. Pepperberg, and F. Bezanilla, Photosensitivity of Neurons Enabled by Cell-Targeted Gold Nanoparticles, Neuron 86, 207 (2015).

[20] N. Martino, P. Feyen, M. Porro, C. Bossio, E. Zucchetti, D. Ghezzi, F. Benfenati, G. Lanzani, and M. R. Antognazza, Photothermal Cellular Stimulation in Functional BioPolymer Interfaces, Sci. Rep. 5, 8911 (2015).

[21] H. Huang, S. Delikanli, H. Zeng, D. M. Ferkey, and A. Pralle, Remote Control of Ion Channels and Neurons through Magnetic-Field Heating of Nanoparticles, Nat. Nanotechnol. 5, 602 (2010).

[22] R. Chen, G. Romero, M. G. Christiansen, A. Mohr, and P. Anikeeva, Wireless Magnetothermal Deep Brain Stimulation, Science 347, 1477 (2015). 
[23] Y. Palti and W. J. Adelman, Measurement of Axonal Membrane Conductances and Capacity by Means of a Varying Potential Control Voltage Clamp, J. Membr. Biol. 1, 431 (1969).

[24] M. Hotka and I. Zahradnik, Membrane Capacitance Changes due to Temperature Increase in Rat Cardiac Myocytes, Biophys. J. 106, 121a (2014).

[25] Y. Jiang, J. L. Carvalho-de-Souza, R. C. S. Wong, Z. Luo, D. Isheim, X. Zuo, A. W. Nicholls, I. W. Jung, J. Yue, D.-J. Liu, Y. Wang, V. De Andrade, X. Xiao, L. Navrazhnykh, D. E. Weiss, X. Wu, D. N. Seidman, F. Bezanilla, and B. Tian, Heterogeneous Silicon Mesostructures for Lipid-Supported Bioelectric Interfaces, Nat. Mater. 15, 1 (2016).

[26] Q. Liu, M. J. Frerck, H. A. Holman, E. M. Jorgensen, and R. D. Rabbitt, Exciting Cell Membranes with a Blustering Heat Shock, Biophys. J. 106, 1570 (2014).

[27] R. D. Rabbitt, A. M. Brichta, H. Tabatabaee, P. J. Boutros, J. H. Ahn, C. C. Della Santina, L. Poppi, and R. Lim, Heat Pulse Excitability of Vestibular Hair Cells and Afferent Neurons, J. Neurophysiol. 116, 825 (2016).

[28] M. Plaksin, E. Kimmel, and S. Shoham, Correspondence: Revisiting the Theoretical Cell Membrane Thermal Capacitance Response, Nat. Commun. 8, 1431 (2017).

[29] M. G. Shapiro, K. Homma, S. Villarreal, C.-P. Richter, and F. Bezanilla, Correspondence: Reply to "Revisiting the Theoretical Cell Membrane Thermal Capacitance Response”, Nat. Commun. 8, 1 (2017).

[30] J. Pan, S. Tristram-Nagle, N. Kucerka, and J. F. Nagle, Temperature Dependence of Structure, Bending Rigidity, and Bilayer Interactions of Dioleoylphosphatidylcholine Bilayers, Biophys. J. 94, 117 (2008).

[31] N. Kučerka, M. P. Nieh, and J. Katsaras, Fluid Phase Lipid Areas and Bilayer Thicknesses of Commonly Used Phosphatidylcholines as a Function of Temperature, Biochim. Biophys. Acta 1808, 2761 (2011).

[32] P. Szekely, T. Dvir, R. Asor, R. Resh, A. Steiner, O. Szekely, A. Ginsburg, J. Mosenkis, V. Guralnick, Y. Dan, T. Wolf, C. Tamburu, and U. Raviv, Effect of Temperature on the Structure of Charged Membranes, J. Phys. Chem. B 115, 14501 (2011).

[33] X. Zhuang, J. R. Makover, W. Im, and J. B. Klauda, A Systematic Molecular Dynamics Simulation Study of Temperature Dependent Bilayer Structural Properties, Biochim. Biophys. Acta 1838, 2520 (2014).

[34] H. Lizhi, K. Toyoda, and I. Ihara, Dielectric Properties of Edible Oils and Fatty Acids as a Function of Frequency, Temperature, Moisture and Composition, J. Food Eng. 88, 151 (2008).

[35] L. G. Gouy, In the Constitution of the Electric Charge on the Surface of an Electrolyte, Compte rendu 149, 654 (1909).

[36] D. L. Chapman, A Contribution to the Theory of Electrocapillarity, Philos. Mag. 25, 475 (1913).

[37] H. O. Stern, For the Theory of the Electrolytic Double Layer, Z. Elektrochem. 30, 508 (1924).

[38] S. Genet, R. Costalat, and J. Burger, A Few Comments on Electrostatic Interactions in Cell Physiology, Acta Biotheoretica 48, 273 (2000).

[39] A. J. Bard and L. R. Faulkner, Electrochemical Methods: Fundamentals and Applications, 2nd ed. (John Wiley \& Sons, New York, 2001).
[40] See Supplemental Material at http://link.aps.org/ supplemental/10.1103/PhysRevX.8.011043 for the suggested model parameters and membrane mobile charge versus subregions potentials curves.

[41] N. Lakshminarayanaiah and K. Murayama, Estimation of Surface Charges in Some Biological Membranes, J. Membr. Biol. 23, 279 (1975).

[42] Y. A. Ermakov, A. Z. Averbakh, A. I. Yusipovich, and S. Sukharev, Dipole Potentials Indicate Restructuring of the Membrane Interface Induced by Gadolinium and Beryllium Ions, Biophys. J. 80, 1851 (2001).

[43] C. C. Valley, J. D. Perlmutter, A. R. Braun, and J. N. Sachs, $\mathrm{NaCl}$ Interactions with Phosphatidylcholine Bilayers Do Not Alter Membrane Structure but Induce Long-Range Ordering of Ions and Water, J. Membr. Biol. 244, 35 (2011).

[44] S. Danner, M. Rappolt, P. Laggner, G. Pabst, A. Hodzic, and S. Janez, Rigidification of Neutral Lipid Bilayers in the Presence of Salts, Biophys. J. 93, 2688 (2007).

[45] O. Szekely, A. Steiner, P. Szekely, E. Amit, R. Asor, C. Tamburu, U. Raviv, and G. Ram, The Structure of Ions and Zwitterionic Lipids Regulates the Charge of Dipolar Membranes, Langmuir 27, 7419 (2011).

[46] P. Martínez and A. Morros, Membrane Lipid Dynamics during Human Sperm Capacitation, Front. Biosci. 1, d103 (1996).

[47] D. C. Sterratt, Q10: The Effect of Temperature on Ion Channel Kinetics, Encycl. Comput. Neurosci. 2551 (2015).

[48] V. Pikov, X. Arakaki, M. Harrington, S. E. Fraser, and P. H. Siegel, Modulation of Neuronal Activity and Plasma Membrane Properties with Low-Power Millimeter Waves in Organotypic Cortical Slices, J. Neural Eng. 7, 045003 (2010).

[49] X. Long, J. Ye, D. Zhao, and S. J. Zhang, Magnetogenetics: Remote Non-Invasive Magnetic Activation of Neuronal Activity with a Magnetoreceptor, Science bulletin 60, 2107 (2015).

[50] M. A. Wheeler, C. J. Smith, M. Ottolini, B. S. Barker, A. M. Purohit, R. M. Grippo, R. P. Gaykema, A. J. Spano, M. P. Beenhakker, S. Kucenas, M. K. Patel, C. D. Deppmann, and A. D. Guler, Genetically Targeted Magnetic Control of the Nervous System, Nat. Neurosci. 19, 756 (2016).

[51] K. Iwasa, I. Tasaki, and R. C. Gibbons, Swelling of Nerve Fibers Associated with Action Potentials, Science 210, 338 (1980).

[52] T. Heimburg and A. D. Jackson, On Soliton Propagation in Biomembranes and Nerves, Proc. Natl. Acad. Sci. U.S.A. 102, 9790 (2005).

[53] A. El Hady and B. B. Machta, Mechanical Surface Waves Accompany Action Potential Propagation, Nat. Commun. 6, 6697 (2015).

[54] B. Krasovitski, V. Frenkel, S. Shoham, and E. Kimmel, Intramembrane Cavitation as a Unifying Mechanism for Ultrasound-Induced Bioeffects, Proc. Natl. Acad. Sci. U.S.A. 108, 3258 (2011).

[55] O. Naor, S. Krupa, and S. Shoham, Ultrasonic Neuromodulation, J. Neural Eng. 13, 031003 (2016).

[56] M. Plaksin, S. Shoham, and E. Kimmel, Intramembrane Cavitation as a Predictive Bio-Piezoelectric Mechanism for Ultrasonic Brain Stimulation, Phys. Rev. X 4, 011004 (2014). 
[57] M. Plaksin, E. Kimmel, and S. Shoham, Cell-Type-Selective Effects of Intramembrane Cavitation as a Unifying Theoretical Framework for Ultrasonic Neuromodulation, eNeuro 3, 1 (2016).

[58] A. Velikonja, E. Gongadze, V. Kralj-Iglic, and A. Iglic, Charge Dependent Capacitance of Stern Layer and Capacitance of Electrode/Electrolyte Interface, Int. J. Electrochem. Sci. 9, 5885 (2014).

[59] F. Bellemare and M. Fragata, Polarity Studies on the Head Group of Single-Layered Phosphatidylcholine- $\alpha$-Tocopherol Vesicles, J. Colloid Interface Sci. 77, 243 (1980).

[60] A. Garreta, S. P. Val-Moraes, Q. García-Fernández, M. Busquets, C. Juan, A. Oliver, A. Ortiz, B. J. Gaffney, I. Fita, A. Manresa, and X. Carpena, Structure and Interaction with Phospholipids of a Prokaryotic Lipoxygenase from Pseudomonas Aeruginosa, FASEB J. 27, 4811 (2013).

[61] D. Marsh, CRC Handbook of Lipid Bilayers (CRC press, Boca Raton, 1990).

[62] H. Oshima, Y. Inoko, and T. Mitsui, Hamaker Constant and Binding Constants of $\mathrm{Ca} 2+$ and $\mathrm{Mg}^{2+}$ in Dipalmitoyl Phosphatidylcholine/Water System, J. Colloid Interface Sci. 86, 57 (1982).

[63] C. Newton, W. Pangborn, S. Nir, and D. Papahadjopoulos, Specificity of $\mathrm{Ca} 2+$ and $\mathrm{Mg} 2+$ Binding to Phosphatidylserine Vesicles and Resultant Phase Changes of Bilayer Membrane Structure, Biochim. Biophys. Acta 506, 281 (1978). 